\title{
EL PRINCIPIO DE LA TRADICIÓN. EUGENIO COSERIU Y LA HISTORIOGRAFÍA LINGÜÍSTICA*
}

\author{
REINHARD MEISTERFELD \\ Universidad de Tubinga
}

1. La profundidad analítica y la coherencia sistemática de la teoría lingüística de Eugenio Coseriu son tales que ese científico, durante más de medio siglo de actividad investigadora, nunca se ha visto obligado a retractar o a sustituir una sola de sus afirmaciones sustanciales y que, aun en lo que se refiere a los rasgos más marginales de su doctrina, son raros los casos en que ha juzgado necesario aplicarles ciertos retoques o modificaciones de formulación. Garantes de esa coherencia y homogeneidad son algunos principios fundamentales de metodología y de epistemología que han guiado su pensamiento lingüístico y sus investigaciones desde los comienzos. Sin embargo, no antes de los últimos lustros de su vida, y, mayormente, en ocasión de los múltiples homenajes que se le han hecho, Eugenio Coseriu ha dado forma explícita a esos principios con el propósito de reconsiderar el conjunto de su obra y de dar cuenta de sus fundamentos y alcances.

Estos principios son cinco, interdependientes y entrelazados, a saber ${ }^{1}$ :

-El principio del realismo o de la objetividad científica, por lo menos como norma ideal;

-El principio del humanismo o del saber originario; es decir que «el saber que el hombre tiene acerca de sí mismo y de sus propias actividades libres» es el fundamento de las ciencias de la cultura;

—El principio de la tradición justificado por ese mismo saber del hablante;

-El principio del antidogmatismo que «concierne a la pluralidad y variedad de las concepciones y orientaciones que se dan en lingüística (y en otras ciencias de la

\footnotetext{
* Permítasenos retomar aquí algunas de las observaciones ya formuladas en nuestra contribución al homenaje que sus amigos de Tubinga han ofrecido a Eugenio Coseriu en ocasión de sus 80 años: Reinhard Meisterfeld, «Eugenio Coseriu und die Geschichte der romanischen Sprachwissenschaft», Adolfo Murguía, ed., Sprache und Welt. Festgabe für Eugenio Coseriu zum 80. Geburtstag, Tubinga, 2002: 141-165.

${ }^{1}$ Nos referimos en particular a la articulación que Eugenio Coseriu ha dado a su cuadro epistemológico de las ciencias del hombre en Eugenio Coseriu, «Discurso pronunciado con motivo de su investidura como doctor honoris causa», Universidad de Granada, ed., Discursos pronunciados en el acto de investidura de doctor «honoris causa», Granada, 1993: 21-35.
}

Odisea, $\mathrm{n}^{\mathrm{0}} 3$, 2003, ISSN 1578-3820, pags. 155-166 
cultura) y recomienda que cada una de ellas se juzgue desde el punto de vista de su coherencia interna»;

—El principio del bien público o de la responsabilidad social.

Aunque, por supuesto, este último se considera el principio ético por excelencia, los otros cuatro tampoco carecen de dimensión ética.

Así, en lo que concierne al principio de la tradición, la ética (la norma deóntica) de la investigación requiere que todo estudioso respete el saber ya alcanzado por las generaciones anteriores. Si es cierto que, hoy en día como siempre, toda exposición de algo «nuevo» en las ciencias suele partir, explícita —o implícitamente-, de «lo que ya se sabía», de «lo que hasta allí pasaba por ser verdad» (u opinión común), no es menos cierto que, en la práctica de la investigación y de sus resultados publicados, la extensión de la memoria histórica se ha ido reduciendo notablemente en estos últimos tiempos ${ }^{2}$. Eugenio Coseriu, por el contrario, ha defendido constantemente la meta ideal de apoderarse de todo el saber acerca del lenguaje que el ingenio humano, a través de los siglos, ha conseguido intuir y formular (siempre que su transmisión hasta el presente no se haya visto impedida por las peripecias históricas). Más aún: sirviéndose de una hermenéutica empatética trata de identificar incluso los contenidos virtuales de las ideas del pasado, apuntando sus desarrollos posibles y su fertilidad futura. Pero el aspecto ético del principio de la tradición no se limita a reclamar la justicia debida a los pensadores del pasado. Por el contrario: ello concierne de igual manera a los empeños de la ciencia actual que, sin perspectiva histórica, serían relegados al olvido inminente $y$, al fin de cuentas, a la futilidad.

El principio de la tradición se funda, por lo tanto, en las normas éticas de la ciencia en general. Sin embargo, en las ciencias de la cultura, el aspecto ético no constituye el único fundamento del principio de la tradición, ni siquiera el más importante. Esto se debe a que, en las ciencias de la cultura, los objetos de estudio resultan íntimamente determinados por su aspecto histórico, más precisamente: los objetos culturales son incluso identificables con su esencia histórica. El principio de la tradición atañe, pues, a la propia epistemología de las ciencias culturales. Tal estado de cosas se debe a las características de los objetos culturales que son productos históricos de la actividad libre y creadora del hombre. Y es aquí que el principio de la tradición se enlaza con el principio del humanismo o del saber originario: todo el saber acerca de los objetos culturales tiene su origen en aquel saber que el hombre tiene acerca de sí mismo y de sus actividades libres. Figurando las lenguas naturales (históricas) entre los objetos culturales, este saber originario, en lingüística, corresponde al saber intuitivo del hablante ${ }^{3}$ :

En las ciencias de la cultura se trata de las actividades libres del hombre, determinadas sólo por su finalidad y no por «causas» (su causa eficiente es simplemente su agente: el hombre). Por ello, en estas ciencias, por un lado, todo planteamiento sólo puede ser "finalista», pues, como nos lo ha enseńado Aristóteles (y han vuelto a enseńárnoslo Vico y, en la lingüística, Pagliaro), ahí donde hay "causa final» (finalidad), no caben planteamientos causales como los que se aplican al mundo de la necesidad, y, por otro lado, estas

\footnotetext{
${ }^{2}$ Cierta reacción a esta pérdida de la memoria histórica está constituida por el creciente interés en los estudios historiográficos de las ciencias de la cultura (y particularmente del lenguaje) que se ha manifestado en los últimos decenios. Sin embargo, tales estudios tienden a aislarse de la actividad investigadora actual y a volverse materia de especialistas.

${ }^{3}$ Citamos a Eugenio Coseriu, «Discurso de investidura», Universidad Autónoma de Madrid, ed., Solemne acto académico con motivo de la investidura como Doctor "Honoris Causa" de los Profesores Carlos Castilla del Pino, Eugenio Coseriu y José Elguero Bertolino, Madrid, 1999: 33-42, p. 36.
}

Odisea, $\mathrm{n}^{\circ} 3,2003$ 
ciencias no pueden fundarse en hipótesis, sino sólo en la certeza del agente de las respectivas actividades (en el verum-certum de Vico) o, mejor, en el saber originario de Husserl: aquel saber que el hombre tiene acerca de sí mismo y de sus actividades libres (y, desde luego, acerca de la finalidad de éstas). De aquí, el principio del saber originario, que en la lingüística es principio del saber intuitivo del hablante.

El cometido de la lingüística consiste, pues, en nada más que en trasladar este saber intuitivo al plano de la reflexión y del saber sistemática y metodológicamente justificable. Y como el hombre dispone de este saber «desde siempre», es posible y aun probable que determinadas cuestiones acerca de la esencia del lenguaje ya hayan sido planteadas y discutidas en el pasado. A la par que de una filosofia perennis se podría, pues, idealmente, hablar de una «perennidad de la lingüística». De ahí que en las ciencias del lenguaje no pueda haber novedad absoluta, sino sólo el conjunto de tradición y novedad. En lo que concierne a su propia teoría lingüística, Eugenio Coseriu defiende, en conformidad con lo dicho, una posición muy modesta en cuanto a la novedad en ella contenida, mas — séanos permitido agregarlo - extremadamente pretenciosa en lo que concierne a su validez (y a la integración de la sabiduría que nos ha legado la tradición $)^{4}$ :

Por ello, en lo que me concierne, estoy siempre dispuesto a admitir que mucho de lo que tiene validez, e incluso todo lo valedero, en mis escritos e investigaciones (en cuanto concepción y método) procede de otros lingüistas y de varios filósofos del lenguaje, a través de un proceso dialéctico de síntesis, cuya base constante de referencia ha sido la realidad misma del lenguaje, tal como se presenta a la introspección reflexiva y a la observación sistemática. Lo cual pienso que no me disminuye en absoluto, ya que sólo significa que, estimulado por formulaciones ajenas, he vuelto a vivir por mi cuenta el mismo paso del saber intuitivo al saber reflexivo y fundado. Y no me cuesta reconocer como maestros ideales, en la lingüística como tal, a Humboldt, a Saussure, a Pagliaro, y, en un plano más alto (el filosófico y epistemológico), a Platón, Aristóteles, Leibniz, Vico, Kant, Hegel, Husserl y Croce, sobre todo a Aristóteles y a Hegel.

Invitándonos a una retrospectiva ontogénica de su teoría, Eugenio Coseriu nos relata, por lo tanto, cómo los maestros ilustres del pasado, a través de un diálogo con su propia cognición viva de la realidad del lenguaje lo han conducido a su concepción. Lo importante de esta afirmación está en que la tradición no puede ser adoptada sin más, sino que ella precisa de la continua coligación con la realidad directamente observada. Más aún: en esta unión de factores la parte decisiva cabe a la cognición de la realidad. Por eso, Coseriu rechaza con firmeza el reproche de servirse eclécticamente de las varias doctrinas de la tradición ${ }^{5}$ :

Una concepción ecléctica, se dirá. No. No se trata de ningún «eclecticismo». No se trata de combinar o de conciliar entre sí teorías o concepciones heterogéneas, sino de conciliarlas con la realidad del lenguaje en lo que tienen de «verdad», como perspectivas diferentes con respecto a «lo mismo", o como parcializaciones diferentes de intuiciones concernientes al mismo conjunto de hechos, y de situar todo problema y todo enfoque en

\footnotetext{
${ }^{4}$ Eugenio Coseriu, «Discurso de investidura» (Madrid), cit., p. 34.

${ }^{5}$ Eugenio Coseriu, «Discurso de investidura» (Madrid), p. 38.
} 
un marco en el que tienen su lugar justificado por la realidad misma del lenguaje: la piedra de toque ha sido siempre para mí esta realidad, tal como se presenta al «saber originario».

2. Con todo eso, Eugenio Coseriu no se da por satisfecho con describir su propio camino intelectual hacia una concepción coherente de la complejidad fenomenológica del lenguaje. Antes bien, a partir de la estructura teórica alcanzada por el proceso dialéctico entre introspección y absorción del saber legado, a la cual él mismo no duda en calificar de «verdad» que «dice las cosas como son», él abre la perspectiva hacia la historia del pensamiento lingüístico en general, en un sentido «filogenético», es decir, la perspectiva de la historiografía lingüística. Que el interés historiográfico de Coseriu no está guiado por simple curiosidad anecdótica sino por su concepción epistemológica de los objetos culturales, lo hemos tratado de mostrar. Lo que nos falta son algunas observaciones sobre su metodología.

Su convicción de disponer de una concepción adecuada de la esencia del lenguaje habilita a Coseriu a deslindar elementos (partes, aspectos) de ella que ya se encuentran en las doctrinas de la tradición. Pero antes de exponer las doctrinas de la tradición a este proceso de discriminación crítica hay que considerarlas con imparcialidad, más aún, con absoluta confianza. Hay que aplicarles el «principio de caridad» de la hermenéutica ${ }^{6}$ :

[... ya de algunos de los profesores que tuve en la Universidad de Iasi, en Rumania, y, sobre todo, de mis grandes maestros italianos (en particular de Antonio Banfi, Antonino Pagliaro y Giovanni Maver) he aprendido el fundamental criterio crítico de la «confianza previa», o sea, he aprendido a no comenzar nunca por negar o rechazar como "falsas» concepciones y tesis formuladas por científicos y pensadores de prestigio y a buscar, en cambio, en cada una de ellas, su núcleo de verdad, entendiendo que toda concepción o tesis formulada por científicos y pensadores auténticos ha de fundarse en alguna intuición certera, a pesar de eventuales desviaciones y parcializaciones en la explicitación de la intuición.

Aplicando con rigurosidad este principio, Coseriu, a menudo, llega a una interpretación extensiva que no se limita a lo que está dicho en los textos explícitamente, sino que abarca también lo que de ello resulta deducible. Y, a veces, por su lectura cuidadosa, consigue despejar los primeros atisbos, en cuanto implícitos, de una nueva concepción más adecuada de la realidad. Así, en su admirable examen de los textos platónicos ${ }^{7}, \operatorname{Coseriu}$ verifica que este filósofo, en general, a pesar de su ingente contribución a la génesis del pensamiento lingüístico occidental, no reconoce la diferencia fundamental entre el significado (en cuanto contenido de las formas fónicas del lenguaje) y los objetos reales designados por los enunciados. Sin embargo, hay dos pasajes en los textos platónicos donde el concepto de «significado» trasluce en la veste de la palabra sovvous, es decir, allí donde se

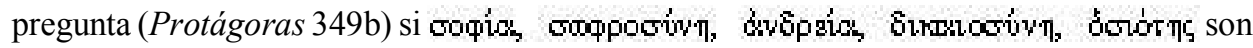

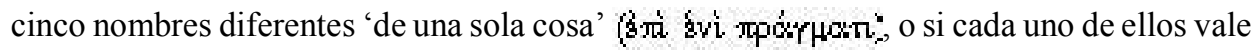

\footnotetext{
${ }^{6}$ Eugenio Coseriu, «Discurso de investidura» (Madrid), p. 34-35.

7 Eugenio Coseriu, «Die sprachphilosophische Thematik bei Platon», Der Physei-Thesei-Streit. Sechs Beiträge zur Sprachphilosophie. Herausgegeben und mit einem Vorwort von Reinhard Meisterfeld (de publicación inminente).
}

Odisea, $n^{\circ} 3,2003$ 


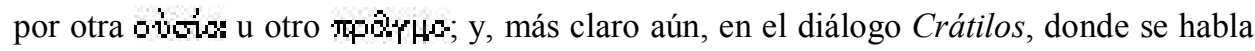

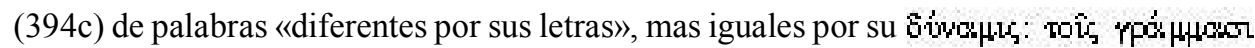

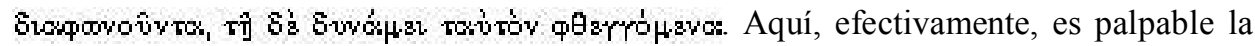

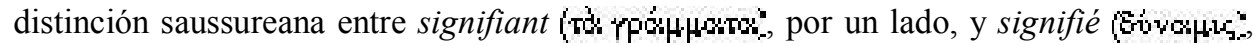
por otro.

Es evidente que una teoría del lenguaje que, como aquélla de Coseriu, tiene sus raíces en el saber originario de los hablantes no puede aceptar una separación arbitraria entre una lingüística «científica» y una lingüística «no científica»o «precientífica» ${ }^{8}$.

Puesto que el fundamento de la lingüística es el saber intuitivo del hablante, es obvio que en la tradición pueden encontrarse intuiciones certeras análogas a las actuales, problemas análogos y soluciones análogas (aunque logradas con otros métodos o sin método ninguno). Y, puesto que todos los lingüistas se fundan en su saber intuitivo de hablantes (aun los que quieren negarlo), es muy posible que enfoques diferentes correspondan a las mismas intuiciones certeras y no sean, en rigor, exclusivos, sino complementarios, siendo sólo parcializaciones diferentes de la misma intuición. Por ello, yo no separo la lingüística llamada «científica» (a partir, por ejemplo, de Bopp) de la lingüística llamada "precientífica»: la diferencia concierne sólo a los métodos, no a los problemas, ni, en rigor, a las soluciones.

El cantus firmus de la historiografía lingüística coseriana es, por ello, la continuidad. Pero se trata de una continuidad ideal, no de una continuidad real de los hechos históricos. Por lo contrario, lo que caracteriza a la historia de la lingüística real son las interrupciones, el cambio de los impulsos y de las perspectivas, las revoluciones (o convulsiones) metodológicas. Y hasta parece que ha sido esta complejidad de la historia de la lingüística la que ha fascinado a Coseriu y que, además del aspecto ético y del epistemológico, lo ha inducido a empeñarse en su investigación.

Fenomenológicamente, las peripecias de la historia de la lingüística, presentan determinadas configuraciones características. Entre ellas, un lugar particular cabe al fenómeno de la emersión. Pues, a pesar de la «perennidad de los problemas y soluciones» $\rangle^{9}$ hay siempre, en el decurso de la tradición conocida, una «primera vez» de su aparición. En el ámbito de las distinciones conceptuales, Coseriu ha conseguido identificar toda una serie de tales «emersiones»:

-la primera distinción entre significado léxico y significado gramatical en el Sofista de Platón;

—la primera distinción entre signifiant y signifié en el De interpretatione de Aristóteles;

- la distinción entre lengua y meta-lengua en el De magistro de S. Agustín;

- la distinción entre sincronía y diacronía en la traducción francesa de François Thurot (1796) del Hermes de James Harris ${ }^{10}$;

- la distinción entre lengua y habla en la Encyclopädie der philosophischen Wissenschaften de Hegel (1817).

\footnotetext{
${ }^{8}$ Eugenio Coseriu, «Discurso de investidura» (Madrid), p. 37.

${ }^{9}$ Eugenio Coseriu, «Discurso pronunciado con motivo de su investidura como doctor honoris causa», 31.

10 James Harris, Hermes: Or, a philosophical inquiry concerning universal grammar, Londres, 1751.
} 
No obstante, el hecho de que un nuevo concepto adecuado haya sido formulado por primera vez no implica necesariamente que éste será acogido por la tradición subsiguiente, sino que, a menudo, él recae en el olvido poco después, dando lugar así a un intervalo en la trasmisión del saber a través de las generaciones. Y puede acontecer que, después de un intervalo, el mismo concepto surja nuevamente, pero sin recuerdo de la tradición pasada, de manera que, en este caso, más propiamente se trata de re-emersión, que de reanudación. Por otro lado, obviamente, también existe el caso de la reanudación (o concatenación) intencional. Pero, a veces, incluso después de su re-emersión, una idea puede ser nuevamente relegada al olvido dando origen a otro intervalo y, por consiguiente, a una secuencia de intervalos. Así, Coseriu ha mostrado cómo la idea de Émile Benveniste, aparentemente original, de que el paradigma de los pronombres personales, en rigor, consiste solo de la primera y de la segunda persona, visto que la tercera persona no es más que una, no-persona» ${ }^{11}$, ya se encuentra, mucho antes, en el Hermes de James Harris que acabamos de citar y, antes de un intervalo todavía más extenso, en Apolonio el Díscolo. Por fin, asimismo, puede darse el caso que determinados conceptos o teoremas que, a primera vista, parecen ser nuevos no constituyen, en realidad, más que el último eslabón de una larga secuencia de testimonios a la cual poco falta para ser considerada como auténtica continuidad de la tradición. Es este el caso del teorema de la arbitrariedad del signo lingüístico, que algunos habían atribuido a la doctrina de Ferdinand de Saussure, pero cuya verdadera historia, desde la antigüedad, ha sido esbozada por Coseriu en un estudio magistral ${ }^{12}$. Además de las emersiones, reanudaciones, secuencias e intervalos existe la filigrana, en parte abierta, pero por la mayor parte cubierta, de las líneas de influjo a través de los espacios y de los tiempos. Uno de los ejemplos más ilustres de tales influencias lo constituye la obra gramaticográfica y lexicográfica de Antonio de Nebrija.

La segunda especie configurativa de los fenómenos de aparición histórica del saber lingüístico son las parcializaciones como facetas y perspectivas. Estas son debidas al hecho de que, en la historia de la lingüística, según los contextos y los intereses diferentes, a menudo, sólo son tematizados (y llevados a una solución), determinados aspectos parciales de un problema o de una cuestión. Así, en el siglo XV, los humanistas, entre otras cosas, ponen en discusión también el problema del cambio lingüístico, pero sólo respecto a la decadencia de la latinidad clásica. Y ya había sido Aristóteles el que había puesto primero y solucionado una de las cuestiones más arduas de la filosofía del lenguaje, a saber: cuál es la esencia del significado lingüístico. Lo caracteriza, en efecto, como tò šv, como unicum, que corresponde a la esencia de las cosas mismas, tal como se presenta a la mente en cuanto

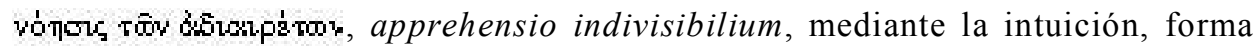
elemental de la cognición. Pero, tácitamente, Aristóteles ha presupuesto que esta primera aprehensión es la misma para todos los hombres. Lo que falta en su teoría es, pues, la faceta

\footnotetext{
${ }^{11}$ Cf. Émile Benveniste, «La nature des pronoms», Morris Halle, Horace G. Lunt, Hugh McLean y Cornelis H. van Schooneveld, eds., For Roman Jakobson, La Haya, 1956: 34-37; y Émile Benveniste, 1966, Problèmes de linguistique générale, París: 251-257.

${ }_{12}$ Eugenio Coseriu, «L'arbitraire du signe. Zur Spätgeschichte eines aristotelischen Begriffes», ASNS 204 (1967): 81-112; v. ea. «L'arbitraire du signe. Sobre la historia tardía de un concepto aristotélico», Eugenio Coseriu, Tradición y novedad en la ciencia del lenguaje. Estudios de historia de la lingüistica, Madrid, 1977: 13-61.
}

Odisea, $n^{\circ} 3,2003$ 
de la diferencia entre las lenguas históricas. A veces, la parcialidad de la cuestión formulada (y la insuficiencia de los resultados) se deben a la falta de los requisitos metodológicos. Así, ya en el siglo XVI, surge la cuestión del origen etimológico de las palabras románicas. Pero las respuestas son, las más de las veces, falsas porque se dan en virtud de la mera semejanza superficial de las palabras. Lo que falta aquí son los métodos históricos y comparativos que no entrarán en la escena de la lingüística antes del siglo XIX.

Las facetas de mayor envergadura pueden llamarse perspectivas. Así, los primeros intentos de relatar el desarrollo histórico de una lengua abren la nueva perspectiva de la Sprachgeschichte, de la «historia de lengua». Con más justo título aún han de considerarse perspectivas las dos orientaciones globales de la lingüística a las cuales se suele llamar diacrónica y sincrónica, respectivamente. Y ha sido Eugenio Coseriu, quien ha mostrado cómo, después del auge del método histórico-comparativo, la vuelta de F. De Saussure hacia la sincronía no es de manera alguna singular y que, por el contrario, en la historia de la lingüística, el cambio de la perspectiva sincrónica y de la diacrónica representa la normali$\operatorname{dad}^{13}$ : de la Antigüedad al Renacimiento la reflexión sobre el lenguaje concierne mayormente a su esencia y a la descripción de sus categorías. El Renacimiento abre el camino a su estudio histórico, aunque se tratase de un primer empeño algo especulativo. La «Gramática General» del siglo XVIII hace resaltar de nuevo el aspecto sincrónico-descriptivo. El siglo XIX, en cambio, dedica casi toda su labor al pasado, mientras que la lingüística moderna y actual, a pesar de su variedad temática y la multitud de sus orientaciones metodológicas, da preferencia a la teoría lingüística y a la lingüística descriptiva.

El tercer tipo de configuraciones en la epifanía histórica del saber lingüístico atañe a la geografía cultural. Ello, porque las peripecias de la historia dan origen a centros preferidos de la evolución del saber lingüístico, núcleos de futuras zonas de irradiación. Así, en la historia de la lingüística románica, el Sur del territorio galorrománico constituye el primer centro de codificación de una variedad romance. Desde el siglo XIII aparecen los primeros tratados gramaticográficos sobre el provenzal (occitano): Lo Donatz Proensals de Uc Faidit y Las Razos de trobar de Raimon Vidal. A partir de este centro hay irradiación hacia Italia y Cataluña. Después del tiempo de los trovadores le toca a Italia emanciparse como centro de la reflexión sobre el romance. Es ahí donde, desde los comienzos del siglo XIV, se cuestiona, por primera vez, científicamente la naturaleza y el origen de las lenguas románicas; $\mathrm{y}$, aún después, durante un período muy extenso, Italia sigue siendo el centro de los primeros estudios de filología románica. En cambio, las primeras tentativas de describir el francés, no se realizan en Francia, como se podría suponer, sino en Inglaterra. En el siglo XVI, hasta cierto punto - a saber: en lo que concierne a la fonética - España puede ser considerada el país dominante de los estudios románicos; mientras que los siglos XVII y XVIII son «siglos franceses» en casi todos los sentidos posibles. A partir de las Observations sur la langue et la littérature provençales de August Wilhelm Schlegel (1818) hasta la última década del siglo XIX, Alemania tiene la reputación de ser el centro de la lingüística románica.

3. Si, por un lado, no cabe duda de que Eugenio Coseriu ha observado el «principio de la tradición» desde los comienzos de sus estudios e investigaciones, es cierto, por el otro,

\footnotetext{
13 Nos referimos en particular a Eugenio Coseriu, Einführung in die Allgemeine Sprachwissenschaft, Tubinga, ${ }^{2} 1992$, cap. 1 (p. 9-25) y a la gráfica en la p. 14.
} 
que la numerosa serie de sus respectivas publicaciones se inicia en sus primeros años de catedrático en Tubinga. Nos contentamos con mencionar los siguientes trabajos ${ }^{14}$ :

1 (37) «François Thurot», ZFSL 77 (1967): 30-34.

2 (39) «L'arbitraire du signe. Zur Spätgeschichte eines aristotelischen Begriffes», ASNS 204(1967): 81-112.

3 (41) «Georg von der Gabelentz et la linguistique synchronique», Word 23 (1967) (= Linguistic Studies Presented to André Martinet, I): 74-100; y Georg von der Gabelentz, Die Sprachwissenschaft, ihre Aufgaben, Methoden und bisherigen Ergebnisse. Durchgesehener Nachdruck der zweiten Auflage von 1901. Herausgegeben und mit einem Vorwort versehen von Gunter Narr und Uwe Petersen, Tubinga, 1969: [5]-[40].

4 (42) «Zur Vorgeschichte der strukturellen Semantik: Heyses Analyse des Wortfeldes 'Schall'», To Honor Roman Jakobson. Essays on the Occasion of His Seventieth Birthday, I, La Haya, 1967: 489-498.

5 (53) «Adam Smith und die Anfänge der Sprachtypologie», Wortbildung, Syntax und Morphologie. Festschrift zum 60. Geburtstag von Hans Marchand, La Haya, 1968:46-54.

6 (69) «Zur Sprachteorie von Juan Luis Vives», Festschrift zum 65. Geburtstag von Walter Mönch, Heidelberg, 1971: 234-255.

7 (71) «Das Problem des Übersetzens bei Juan Luis Vives», Interlinguistica. Sprachvergleich und Übersetzung. Festschrift zum 60. Geburtstag von Mario Wandruszka, Tubinga, 1971: 571-582.

8 (75) «Über die Sprachtypologie Wilhelm von Humboldts. Ein Beitrag zur Kritik der sprachwissenschaftlichen Überlieferung», Beiträge zur vergleichenden Literaturgeschichte. Festschrift für Kurt Wais zum 65. Geburtstag, Tubinga,1972: $107-135$.

9 (77) «Las etimologías de Giambullari», en: Thomas T. Büttner, ed., Homenaje a Antonio Tovar, Madrid, 1972: 95-103.

10 (96) Sprache und Funktionalität bei Fernão de Oliveira (1536), Lisse, 1975; con el título «'Taal en functionaliteit' bei Fernão de Oliveira» también Ut Videam: Contributions to an Understanding of Linguistics. For P. Verburg on the Occasion of His 70th Birthday, Lisse, 1975: 67-90.

11 (97) «Andrés de Poza y las lenguas de Europa», Studia hispanica in honorem R. Lapesa, III, Madrid, 1975: 199-217.

12 (98) «Andreas Müller und die Latinität des Rumänischen», RRL 20, 4 (1975) [= Homenaje a A. Graur]: 327-332.

\footnotetext{
${ }^{14}$ Los números entre paréntesis se refieren a la lista «Verzeichnis der Schriften Coserius», Johannes Kabatek, Adolfo Murguía, »Die Sachen sagen, wie sie sind...«. Eugenio Coseriu im Gespräch, Tubinga, 1997, p. 271-305.
}

Odisea, $n^{\circ} 3,2003$ 
13 (99) «Die rumänische Sprache bei Hieronymus Megiser (1603)», SCL 26, 5 (1975) [= Homenaje a A. Rosetti]: 473-480.

14 (104) Prefacio a: J. Albrecht, ed., Pierre-Nicolas Bonamy. Vier Abhandlungen zum Vulgärlatein und zur Frühgeschichte des Französischen, Tubinga, 1975: 1-4.

15 (108) «Zur Kenntnis der rumänischen Sprache in Westeuropa im 16. Jahrhundert (Genebrard und Andrés de Poza)», Scritti in onore di Giuliano Bonfante, II, Brescia, 1976:527-545.

16 (109). «Stiernhielm, die rumänische Sprache und das merkwürdige Schicksal eines Vaterunsers», Romanica 8, (1975) (=Estudios dedicados a D. Gazdaru, IV ), La Plata, 1976: 7-23.

17 (110).«Un germanista vizcaíno en el siglo XVI. Andrés de Poza y el elemento germánico del español», Anuario de Letras, México, 13 (1975) [1976]: 5-16.

18 (111) «Das Rumänische im ‘Vocabolario’ von Hervás y Panduro», ZRPh 92 (1976): $394-407$.

19 (123) «Vulgärlatein und Rumänisch in der deutschen Tradition», Homenaje a Rodolfo Grossmann, Francfort (del Meno), 1977: 337-346.

20 (125) «Zur Kenntnis des Rumänischen in Westeuropa um 1600. Megiser und Botero», CL 22 (1977) [= Homenaje a D. Macrea]: 151-153.

21 (126) «Rumänisch und Romanisch bei Hervás y Panduro», Dacoromania. Jahrbuch für östliche Latinität 3, (1975-76) [1977]: 113-134.

22 (137) «Hervás und das Substrat», SCL 29, 5 (1978) [= Homenaje a I. Iordan]: 523-530.

23 (138) «Lo que se dice de Hervás», Estudios ofrecidos a Emilio Alarcos Llorach, III, Oviedo, 1978: 35-58.

24 (154) «Humboldt und die moderne Sprachwissenschaft», Arnold $\underset{\text { C } i k o b a v a s}{\mathrm{~V}}$ (dabadebis 80 c'listavisadmi midzghvnili k'rebuli) [=Homenaje a A. Cikobava], Tiflis, 1979: 20-29.

25 (163) «Un précurseur méconnu de la syntaxe structurale: H. Tiktin», Recherches de linguistique. Hommages à Maurice Leroy, Bruselas, 1980: 48-62.

26 (165) «Griselini, das Rumänische und das Vulgärlatein», Stimmen der Romania. Festschrift für W. Th. Elwert zum 70. Geburtstag, Wiesbaden, 1980: 537-549.

27 (167).«Sardica ut in oppidis», Herbert J. Izzo (Hg.), Italic and Romance. Linguistic Studies in Honor of Ernst Pulgram, Amsterdam, 1980 (= Current Issues in Linguistic Theory 18): 317-326.

28 (187). «Zu den Etymologien von Miron Costin», Kurier der Bochumer Gesellschaft für rumänische Sprache und Literatur, 12, (1981): 8-15.

29 (219) «Megiser und Trubar», Simpozij Slovenci v evropski reformaciji. Zbornik prispevkov, Ljubljana, 1986: 51-56. 
30 (220) «Gli 'antenati’ di Ascoli», G. I. Ascoli. Attualità del suo pensiero a 150 anni dalla nascita (= Atti del XIII Incontro Culturale Mitteleuropeo. Gorizia, 24-25 novembre 1979), Florencia, 1986: 21-36.

31 (227) «Theodor Gartners Werk im Bereich der Rumänistik», Akten der Theodor Gartner-Tagung (Rätoromanisch und Rumänisch) in Vill/Innsbruck 1985 (Romanica Aenipontana, XIV), (ed. G. A. Plangg y M. Iliescu) Innsbruck, 1987: 277287.

32 (288) «La 'découverte' des voyelles nasales», R. van Deyck, ed., Diachronie et variation linguistique. Les nasalisations dans le monde roman, (=Communication \& Cognition, 27, 1-2), Gante, 1994: 7-19.

33 (294) «Von den universali fantastici», Jürgen Trabant, ed., Vico und die Zeichen. Vico e $i$ segni, Tubinga, 1995: 73-80.

34 (298) «Das westeuropäische Bild vom Südslawischen zur Zeit Primus Trubers», Rolf-Dieter Kluge, ed., Ein Leben zwischen Laibach und Tübingen. Primus Truber und seine Zeit, Munich, 1995: 174-185.

35 (337) «Andrés de Poza, lingüista del Renacimiento», Mikel Gorrotxategi, Henrike Knörr (Hgg.), Actas de las II Jornadas de Onomástica Orduña, Septiembre de 1987. II. Onomastika Jardunaldien Agiriak Urduña, 1987ko Iraila, Bilbao, 2000, (=Onomasticon Vasconiae 17): 31-77.

36 (351) «El lugar de los universales fantásticos en la filosofía de Vico», E. Hidalgo Serna, M. Marassi, J. M. Sevilla, J. Villalobos, (eds.), Pensar para el nuevo siglo. Vico y la cultura europea, vol. 1, Lenguaje, poética y retórica filosófica, Nápoles, 2001:3-22.

Y las colecciones:

37 (116) Tradición y novedad en la ciencia del lenguaje, Madrid, 1977, (con los núme$\operatorname{ros} 2,6,7,9,5,1,8$ y 9 de nuestra lista en versión española).

38 (177) Von Genebrardus bis Hervás, Tubinga, 1981 (=Lingua et Traditio 2), (que contiene, entre otros, los trabajos 1, 13, 20, 16, 12, 26, 18 y 21 de nuestra lista.

Además, se podrían citar varios trabajos que se refieren a la lingüística moderna y actual y algunos otros con una temática más específicamente filosófica, de manera que se impone la conclusión de que las investigaciones de Coseriu en el ámbito de la historiografía lingüística son impresionantes por su número y por su variedad. Sin embargo, se podría preguntar, si Coseriu, visto el volumen de sus lecturas y su capacidad de integración no ha intentado recoger sus escrutinios históricos en una obra historiográfica de síntesis. A esta pregunta se puede responder que sí, que Coseriu, efectivamente, ha reunido su trabajo de investigación en una obra sintética, más exactamente, incluso en varias grandes obras sintéticas de historiografía lingüística. Sólo que éstas no han sido publicadas todavía, hecho que tienen en común con gran parte de la obra coseriana; o, mejor dicho, no se han hecho públicas si no por la enseñanza oral de su ilustre autor. Existen, en realidad, entre los materiales del Archivo Coseriu, los manuscritos de dos grandes ciclos de cursos intitulados 'Historia de la lingüís- 
tica románica' e 'Historia de la filosofía del lenguaje', dados ambos entre 1970 y 1976. Además de esto hay manuscritos de cursos semestrales sobre varias secciones y corrientes de la lingüística moderna y actual. Es verdad que numerosos cursos de Coseriu han sido publicados, de forma más o menos provisional, en base a los apuntes de sus discípulos. ${ }^{15}$ Así, por ejemplo, la primera parte de la «Geschichte der Sprachphilosophie» ['Historia de la filosofía del lenguaje'] ha tenido luego un éxito significativo a pesar de la insuficiencia excusable de los dos cuadernos publicados por Gunter Narr y Rudolf Windisch en 1969 y en 1972. No obstante, este texto acaba de encontrar ahora, aproximadamente, su planeada forma de manual gracias a su elaboración y actualización bibliográfica realizada, aún en diálogo con Coseriu, por Jörn Albrecht, su discípulo y catedrático en Heidelberg ${ }^{16}$, que se ha empeñado en presentarnos, en un futuro no demasiado lejano, también el segundo volumen. Menos privilegiada ha sido la suerte de la 'Historia de la lingüística románica'. En efecto, Eugenio Coseriu había dedicado a esta temática, en los años setenta, un ciclo de no menos de seis cursos semestrales en los que trataba el desarrollo del pensamiento lingüístico romance desde los comienzos hasta el siglo XIX y, además, había manifestado su intención de establecer, en el ámbito de su actividad docente en Tubinga, un centro de investigación histórica de la lingüística románica. No obstante, esta labor no excedió nunca el estrecho radio de efectividad de la enseñanza oral de su autor y, salvo algunos reflejos en las publicaciones de sus discípulos ${ }^{17}$, durante tres decenios, no formó parte de la obra coseriana accesible. Fue este inconveniente, el que motivó, en 1998, a Brigitte Schlieben-Lange y Johannes Kabatek, los instigadores y directores del proyecto Eugenio Coseriu patrocinado por la Deutsche Forschungsgemeinschaft a escoger la primera parte de este ciclo como ensayo de una futura elaboración de la obra coseriana manuscrita. Entretanto, el resultado de este primer ensayo ha sido publicado ${ }^{18}$. La línea directriz de la redacción ha sido, por un lado, mantener la contextura del manuscrito coseriano, a saber, sus principios de selección, de discriminación y de evaluación de los materiales históricos, pero, por el otro, precisarlo y actualizarlo en sus aspectos técnico y bibliográfico y ampliar su sustancia descriptiva ${ }^{19}$. En cuanto a los pormenores de la elaboración cf. Reinhard Meisterfeld, «Eugenio Coseriu und die Geschichte der romanischen Sprachwissenschaft», (2002) op. cit.

No cabe duda de que esta abertura de un discurso historiográfico de Coseriu en continuidad es motivo de satisfacción. Pero también es evidente que no se puede tratar más que de un primer paso, porque de la totalidad del material sobre la historia de la lingüística románica que abarca más de 2500 medias páginas manuscritas han sido elaboradas menos

\footnotetext{
${ }^{15}$ Cf. la lista de las «Vorlesungsnachschriften», Johannes Kabatek, Adolfo Murguía, »Die Sachen sagen, wie sie sind...«, 306-308.

${ }^{16}$ Eugenio Coseriu, Geschichte der Sprachphilosophie. Von den Anfängen bis Rousseau. Neu bearbeitet und erweitert von Jörn Albrecht. Mit einer Vor-Bemerkung von Jürgen Trabant, Tubinga - Basilea, 2003. ${ }^{17}$ Cf. (como ejemplo más reciente): Jens Lüdtke, «Diachrone romanische Sprachwissenschaft und Sprachgeschichtsschreibung / Linguistique romane diachronique et histoire des langues romanes», Günter Holtus, Michael Metzeltin, Christian Schmitt (eds.), Lexikon der Romanistischen Linguistik, vol. I, 1: Geschichte des Faches Romanistik. Methodologie (Das Sprachsystem), Tubinga, 2001: 1-35 (= § 1: «Romanische Philologie von Dante bis Raynouard», sección a.).

${ }^{18}$ Eugenio Coseriu / Reinhard Meisterfeld, Geschichte der romanischen Sprachwissenschaft. Vol. 1: Von den Anfängen bis 1492, Tubinga, 2003.

19 En cuanto a los pormenores de la elaboración cf. Reinhard Meisterfeld, «Eugenio Coseriu und die Geschichte der romanischen Sprachwissenschaft», (2002) op. cit.
}

Odisea, $n^{\circ} 3,2003$ 
de cien. Y, aunque el primer volumen no pueda ser considerado, en lo que concierne a la relación entre el material manuscrito y el texto publicado, como representativo de la obra entera - porque las notas de Coseriu, en esta primera fase de su intento, aún demuestran el carácter de esbozo - es natural que la elaboración de la parte restante constituye una tarea ingente. Pero hay que integrar ésta, a su vez, en el cometido mayor de tornar disponible el conjunto de la obra manuscrita de Coseriu que excede la publicada por su dimensión. En realidad, este científico excepcional, maestro en un sentido de venerable antigüedad, ha siempre concebido su doctrina, en primer lugar, como enseñanza directa, como alocución a un público presente. En lo que respecta a nosotros, es la deontología de las ciencias la que nos impone conferirle subsistencia y legarla a las generaciones posteriores por el principio de la tradición. 